\title{
FIVE-LEVEL NON-SEQUENTIAL THIRD-ORDER RESPONSE SURFACE DESIGNS AND THEIR EFFICIENCIES
}

\author{
Iwundu M. P. and Agadaga G. $O$. \\ Department of Mathematics and Statistics, University of Port Harcourt, Nigeria \\ mary.iwundu@uniport.edu.ng,gregagadaga@gmail.com
}

Cite this article:

Iwundu M. P., Agadaga G. O. (2022), Five-level Non-

Sequential Third-Order

Response Surface Designs and their Efficiencies. African

Journal of Mathematics and

Statistics Studies 5(1), 14-32. DOI: 10.52589/AJMSSIJYZJB2C.

\section{Manuscript History}

Received: 16 Nov 2021

Accepted: 1 Dec 2021

Published: 3 Feb 2022

Copyright $($ ) 2022 The Author(s). This is an Open Access article distributed under the terms of Creative Commons AttributionNonCommercial-NoDerivatives 4.0 International (CC BY-NC-ND 4.0 ), which permits anyone to share, use, reproduce and redistribute in any medium, provided the original author and source are credited.
ABSTRACT: New non-sequential third-order response surface designs are proposed with good optical properties. They are suitable as one-stage experimental designs for use in modeling third-order effects. The new designs are presented for cuboidal region in $k$ dimensions and the technique employed in the construction of the non-sequential designs on the cuboidal region is flexible for use in regions that may be non-cuboidal. The new non-sequential designs lay importance on the use of axial points and two or three other blocks of points selected from a discrete design region such that the design is non-singular. For a continuous design region, uniform grids are formed over the entire design region. Five grid levels are utilized in this work thus resulting in $5^{k}$ grid points from which blocks of points are selected to form the desired non-sequential designs. The goodness of the designs is assessed via optimality and efficiency criteria and the new designs possess good optimality properties and are very high by G-efficiency.

KEYWORDS: Non-Sequential designs, Cuboidal region, Uniform grids, Design optimality and G-efficiency. 


\section{INTRODUCTION}

Many authors have researched response surface designs particularly for first- and second-order response surfaces. A vast number of literatures exist to their credits and have been wonderful foundations for research works in Response Surface Methodology (RSM) involving process control and optimization. Response Surface Methodology has attracted a consistently growing interest among researchers who find its applicability in a wide range of study areas including Biological, Chemical, Agricultural, Pharmaceutical, Engineering, Food Science, etc. The goal of Response Surface Methodology is primarily to investigate the relationship between the controllable variables or process factors and the quality characteristic of a product. Foundationally, Response Surface Methodology was proposed by Box and Wilson (1951) and improved upon by works of Box (1952, 1954), Box and Behnken (1959). Response Surface Methodology which uses statistical and mathematical techniques has continued to play the vital role in developing, optimizing and improving processes involving several input variables, $\xi_{1}, \xi_{2}, \cdots, \xi_{k}$ which potentially influence some performance measure or quality characteristic, $y$, of the process under study. It is worthy to note that the form of the relationship between the controllable variables or process factors and the quality characteristic of a product is usually unknown and may only be approximated by a suitable polynomial, popularly considered of first-or second-order.

The relationship between the input variables and the response variable may be expressed as

$y=f\left(\xi_{1}, \xi_{2}, \cdots, \xi_{k}\right)+\varepsilon$

where $\varepsilon$ is the random error component assumed to have a normal distribution with zero mean and constant variance such that

$E(y)=f\left(\xi_{1}, \xi_{2}, \cdots, \xi_{k}\right)=\eta$

For convenience, the natural input variables $\xi_{1}, \xi_{2}, \cdots, \xi_{k}$ would usually be transformed to dimensionless coded variables $x_{1}, x_{2}, \cdots, x_{k}$. When it is assumed that there is little or no indication of the presence of curvature in the response function and no interaction between the input variables, a first-order effects model is considered an adequate approximating polynomial. However, if there is an indication of interaction between the independent variables, the first-order model with a measure of interaction is utilized. Model inadequacy is seen to exist when there is substantial curvature in the true response function (indicated by the lack of fit of first-order model) and the need for an approximating polynomial of higher order arises. Most researchers find polynomials of second order giving a better representation of the unknown functional form in the presence of lack of fits of first-order models. Myer et al. (2009) suggested exploring higher order models in the presence of lack of fits of second-order models.

Third-order response surface designs are becoming a growing trend as recent literature suggests experimental situations involving second-order lack of fits. Like all RSM approximating functions, the third-order model is used for approximating the unknown response function that is assumed to contain cubic effects. The third-order model consists of the first-order terms, cross product terms, all the quadratic terms, cross products with the quadratic terms and the cubic terms. Generally, when the dth-order model appears insufficient to describe the true existing relationship between the response of interest and the predictor variables due to the 
presence of higher terms or lack of fit, then a $(d+1)$ th-order is required to fit the model adequately. In terms of the coded input variables, the third-order model is given by the function

$$
\begin{aligned}
\eta=\beta_{0}+\sum_{i=1}^{k} & \beta_{i} x_{i}+\sum_{i<j=2}^{k} \beta_{i j} x_{i} x_{j}+\sum_{i=1}^{k} \beta_{i i} x_{i}^{2}+\sum_{i<j=2}^{k} \beta_{i j j} x_{i} x_{j}^{2} \\
& +\sum_{i<j=2}^{k} \beta_{i i j} x_{i i}^{2} x_{j}+\sum_{i<j<l=3}^{k} \beta_{i j l} x_{i} x_{j} x_{l}+\sum_{i=1}^{k} \beta_{i i i} x_{i i i}^{3} \\
& +\varepsilon
\end{aligned}
$$

which contains $\left(\frac{k+3}{3}\right)$ model parameters. The $\beta^{\prime} s$ are unknown parameters of the model and may be estimated using the method of least squares. The estimated response, in matrix form, is given as

$\hat{y}=X \hat{\beta}$

where the least squares estimate of vector of the unknown model parameters $\beta$ is

$\hat{\beta}=\left(X^{\prime} X\right)^{-1} X^{\prime} y$

Conventionally, the unknown parameters are estimated on the basis of $N$ uncorrelated observations. The vector $y$ denotes the vector of observations and the matrix $X$ denotes the model matrix. From the least squares estimates of the unknown parameters, the estimated response is

$\hat{y}=X\left(X^{\prime} X\right)^{-1} X^{\prime} y=H y$

where $H=X\left(X^{\prime} X\right)^{-1} X^{\prime}$ is called the hat matrix and puts the "hat" on the vector of fitted or estimated value. The diagonal elements of the hat matrix shall form the basis for the construction of the new non-sequential designs.

According to Kahng (2007) the diagonal elements of the hat matrix are regarded as one of the basic components of influence in linear regression models. Each diagonal element $h_{i i}$ of the hat matrix is called a leverage and measures the extent to which the fitted regression model $\hat{y}_{i}$ is attracted by the given observation or data point $y_{i}$. In essence, the $i^{\text {th }}$ leverage $h_{i i}$ quantifies the influence that the observation $y_{i}$ has on its predicted value $\hat{y}_{i}$. The diagonal elements of the hat matrix are such that $0 \leq h_{i i} \leq 1$ and $\sum_{i=1}^{N} \quad h_{i i}=p$; where $N$ is the number of data points and $p$ is the number of model parameters, including the intercept. In this work we seek to generate new non-sequential third-order response surface designs that possess some good optimality properties. For illustrative purposes, the generation of non-sequential third-order designs in two or three design variables shall be considered. A technique using principles of Hat matrix is adopted in this work and offers non-sequential designs that are optimally efficient for third-order models. The new designs are practically viable to implement in various fields of study regardless of the number of control variables. 


\section{Review on Some Third-Order Response Surface Designs}

Although most researchers over time had concentrated on designs of first and second order with particular interest in design rotatability, Bose and Draper (1958) presented several methods for obtaining rotatable designs involving second and third order in three and higher dimensions. Gardiner et al. (1959) considered third-order rotatable designs for exploring response surfaces. Specifically, third-order rotatable designs for two and three factors were obtained and moment and non-singularity conditions for third-order rotatability were derived. Draper (1960a) constructed third-order rotatable designs in three dimensions by combining pairs of second-order rotatable designs. Draper (1960b) considered the construction of thirdorder rotatable design in four dimensions and gave moment conditions for which a set of points would form a third-order rotatable design in $k$ dimensions.

Das (1961) obtained some new third order rotatable designs up to eight factors. Das and Narasimham (1962) constructed third order rotatable designs both sequentially and nonsequentially, up to fifteen factors, using Doubly Balanced Incomplete Block Designs and complementary Balanced Incomplete Block Designs. Anjaneyulu et al. (1994-1995) constructed third-order slope rotatable designs using doubly balanced incomplete block designs. Mutiso (1998) constructed specific and sequential second- and third-order rotatable designs in $\mathrm{k}$ dimensions. Anjaneyulu et al. (2001) introduced third-order slope rotatable designs using central composite type design points.

Practically, if the fit of second-order response is inadequate for the design points, experiment must be continued so as to fit a third-order response surface (Seshu Babu et al. 2015). Castillo et al. (2004), Gao et al. (2009), Norulaini et al. (2009) and Arshad et al. (2012) have documented practical situations where the second-order lack of fits arises in experimental situations. Erickson (2007) in his work on wind tunnel experiments saw the need to use higher order models for proper characterization of nonlinear aerodynamic forces encountered due to interacting shock waves. Landman et al. (2007) considered a wind-tunnel testing problem to establish high performance aircraft's aerodynamic behaviour using Response Surface Methodology approach involving third-order response surface designs. The need arose when the classical second-order central composite design showed inadequacy in prediction qualities. Hence, Landman et al. (2007) developed a hybrid third-order design called a Nested Face Centered Design (NFCD) for the purpose of the investigation.

Yang (2008) generated many third-order designs either using principles of nesting or augmentation. Yang's third-order designs include Nested Face Centered Design (NFCD), Full third-order I-optimal design (FTOD), CCD Augmented by I-optimal (CCDA) and Latin Hypercube Space Filled Design Augmented by I-optimal design (LHDA). Seshu babu et al. (2014) constructed Third-Order Slope Rotatable Designs (TOSRD) from principles of Balanced Incomplete Block Designs (BIBD). In view of wide applicability of third-order models in RSM and importance of slope rotatability, Seshu Babu et al. (2015) introduced Cubic Slope Rotatable Designs Using Balanced Incomplete Block Design in four dimensions and showed that Symmetry assumptions for third-order slope rotatability was satisfied.

Owing to the fact that RSM has applicability in many experimental fields such as Agriculture, Human Medicine, Veterinary Science, Aerodynamics, Meteorological, Biological, Pharmaceutical and Chemical Industry (See Seshu Babu et al. 2015), much interest has been shown in the development of third-order response surface methodology as a way of combating 
problems associated with lack of fits of second-order models, in experimental situations. Baker and Bargmann (1985) applied orthogonal central composite designs of the third-order in the evaluation of sensitivity and plant growth simulation. They considered the effect of using orthogonal cubic response surfaces against response surfaces and found out that orthogonal cubic surfaces provided insight into higher order relationships and further provided a measure of the relative sensitivity of yield to experimentally determined parameter values. Nyakundi and Matunde (2019) presented a hypothetical sequential third-order rotatable design in four Dimensions by combining two four-level second-order rotatable designs. An experimental design was set up to investigate the effects of five fertilizer ingredients on the yield of hybrid maize in Trans-Nzoia using the sequential third-order rotatable design in four dimensions under field conditions. Also, Nyakundi (2019) presented a hypothetical sequential third-order rotatable design in five Dimensions by combining two five-level second-order rotatable designs. An experimental design was set up to investigate the effects of five fertilizer ingredients on the yield of hybrid maize in Trans-Nzoia using the sequential third-order rotatable design in five dimensions under field conditions.

\section{Non-sequential Designs}

In design construction, sequential and non-sequential procedures are often encountered. The general concept of sequential designs in Response Surface Methodology was considered by Box and Wilson (1951) and requires that experimental points are moved in a sequential manner along the gradient-based direction. The popularly encountered second-order Central Composite Design is one of such designs that are constructed in a sequential manner by augmentation of a first-order design. Non-Sequential designs on the other hand constitute experimental designs that are carried out at a single stage. Cheng and Wu (2001) investigated non-sequential designs and revealed that saving experimentation time and run size is an advantage of running a onestage experiment. Such a non-sequential experimental approach seems quite practicable in agricultural settings where experiments require long periods of waiting between various stages to the extent that the results of single experimentation may be the only ones available as against having a series of experiments gotten through a sequential process (See Khuri, 2017). Hence unlike sequential designs that require various stages of experimentation, non-Sequential designs constitute experimental designs that are carried out only at a single stage. The threelevel Box-Behnken design due to Box and Behnken (1960) is a typical example of a nonsequential second-order design.

Sanchez and Sanchez (2005) proposed a non-sequential design that focuses primarily on the second-order terms with the aim of reducing the number of experimental runs of a central composite design. Alaeddini et al. (2013b) noted that the designs by Sanchez and Sanchez (2005) and the Box and Behnken design are not adaptive. In a comparative study on sequential and non-sequential designs carried out by Dette and Kwiecien (2004) for discriminating between nested regression models, it was observed that higher probability was associated with non-sequential designs than with sequential methods. Although non-sequential designs do not guarantee achieving the best possible efficiency in the appropriate model, it was observed using simulation study that for realistic sample sizes the efficiencies of the non-sequential designs for the estimation of the parameters in an appropriate model are at least as high as the corresponding efficiencies of the sequential methods. 


\section{Rules for Non-Sequential Generation of New Third-Order Designs}

Given a response function $y$ that is influenced by a set of independent variables $x_{1}, x_{2}, \ldots, x_{k}$ and it is suspected that a third-order model would adequately establish the relationship between $y$ and the $x_{i}$ 's, we seek an efficient non-sequential design for the model. If the design region is discrete, having a finite number of support points, the total number of such points shall make up $\widetilde{N}$ admissible candidate points. However, for a continuous design region, we adopt the formation of uniform grids over the entire region. The resulting grid points constitute the $\widetilde{N}$ admissible candidate points. For the purpose of this research work, five grid levels $[-1,-1 / 2,0$, $1 / 2,1]$ are chosen and lead to the generation of grid points that make up $\widetilde{N}$ on a cuboidal design region. The choice of the five grid levels is such that yields uniform grids and allows manageable size of candidate points to be used in the design construction. Furthermore, it allows candidate points to spread over the entire design space.

The rules that govern the generation of the new non-sequential designs as follows;

i. $\quad$ Form $5^{\mathrm{k}}$ grid points on the given region.

ii. Obtain the model matrix X using the grid points and the model.

iii. Select three or four blocks of points with maximum $h_{i i}$ entries. The axial points are essential for optimality of the new third-order non-sequential third-order design.

iv. The arrangement of the design points giving rise to the maximum determinant value of the information matrix yields the desired Non-Sequential Third-Order Design.

Using the $\widetilde{N}$ candidate points, the elements of the model matrix for the third-order model are as defined in the matrix $X$.

$$
X=\left[\begin{array}{ccccccccccccc}
1 & x_{11} & x_{12} & \cdots & x_{1 k} & x_{11} x_{12} & x_{11} x_{13} & \cdots & x_{1 k-1} x_{1 k} & \cdots & x_{11}^{2} & \cdots & x_{1 k}^{3} \\
1 & x_{21} & x_{22} & \cdots & x_{2 k} & x_{21} x_{22} & x_{21} x_{23} & \cdots & x_{2 k-1} x_{2 k} & \cdots & x_{21}^{2} & \cdots & x_{2 k}^{3} \\
1 & x_{31} & x_{32} & \cdots & x_{3 k} & x_{31} x_{32} & x_{31} x_{33} & \cdots & x_{3 k-1} x_{3 k} & \cdots & x_{31}^{2} & \cdots & x_{3 k}^{3} \\
\vdots & \vdots & \vdots & \vdots & \vdots & \vdots & \vdots & \cdots & \vdots & \vdots & \vdots & \vdots & \vdots \\
1 & x_{\widetilde{N} 1} & x_{\tilde{N} 2} & \cdots & x_{\tilde{N} k} & x_{\widetilde{N} 1} x_{\widetilde{N} 2} & x_{\widetilde{N} 1} x_{\widetilde{N} 3} & \cdots & x_{\widetilde{N} k-1} x_{\widetilde{N} k} & \vdots & x_{\widetilde{N} 1}^{2} & \vdots & x_{\widetilde{N} k}^{3}
\end{array}\right]
$$

The associated hat matrix is an $\widetilde{N} \times \widetilde{N}$ matrix whose diagonal elements $h_{i i}$ are such that

$0<h_{i i}<1$ and $\sum_{i=1}^{N} \quad h_{i i}=p$

Blocks of support points are formed by the values of the diagonal elements of the hat matrix. Specifically, the diagonal elements $h_{i i}$ of the hat matrix are arranged according to their magnitudes and support points that have the same $h_{i i}$ values are grouped together to form various blocks from which the new non-sequential third-order designs will be based.

The goodness of the new designs shall be examined using optimality and efficiency measures. A determinant-based method as well as a variance-based method shall be employed in the assessment of the design's goodness. 


\section{Non-Sequential third-order response surface designs in $k$ control variables}

The analysis involved in the construction of non-sequential third-order response surface designs is presented for $k=2$ and 3 design variables. The case of the non-sequential design construction requires different blocks of points being combined to generate the required design at one occasion.

\section{Analysis involving two design variables}

For $k=2$, the full parameter response surface model is given as

$y=\beta_{0}+\beta_{1} x_{1}+\beta_{2} x_{2}+\beta_{12} x_{1} x_{2}+\beta_{11} x_{1}^{2}+\beta_{22} x_{2}^{2}+\beta_{122} x_{1} x_{2}^{2}+\beta_{112} x_{11}^{2} x_{2}+\beta_{111} x_{111}^{3}+$

$\beta_{222} x_{222}^{3}+\varepsilon$

and contains 10 model parameters. The model matrix corresponding to the $5^{k}=25$ grid points is given as

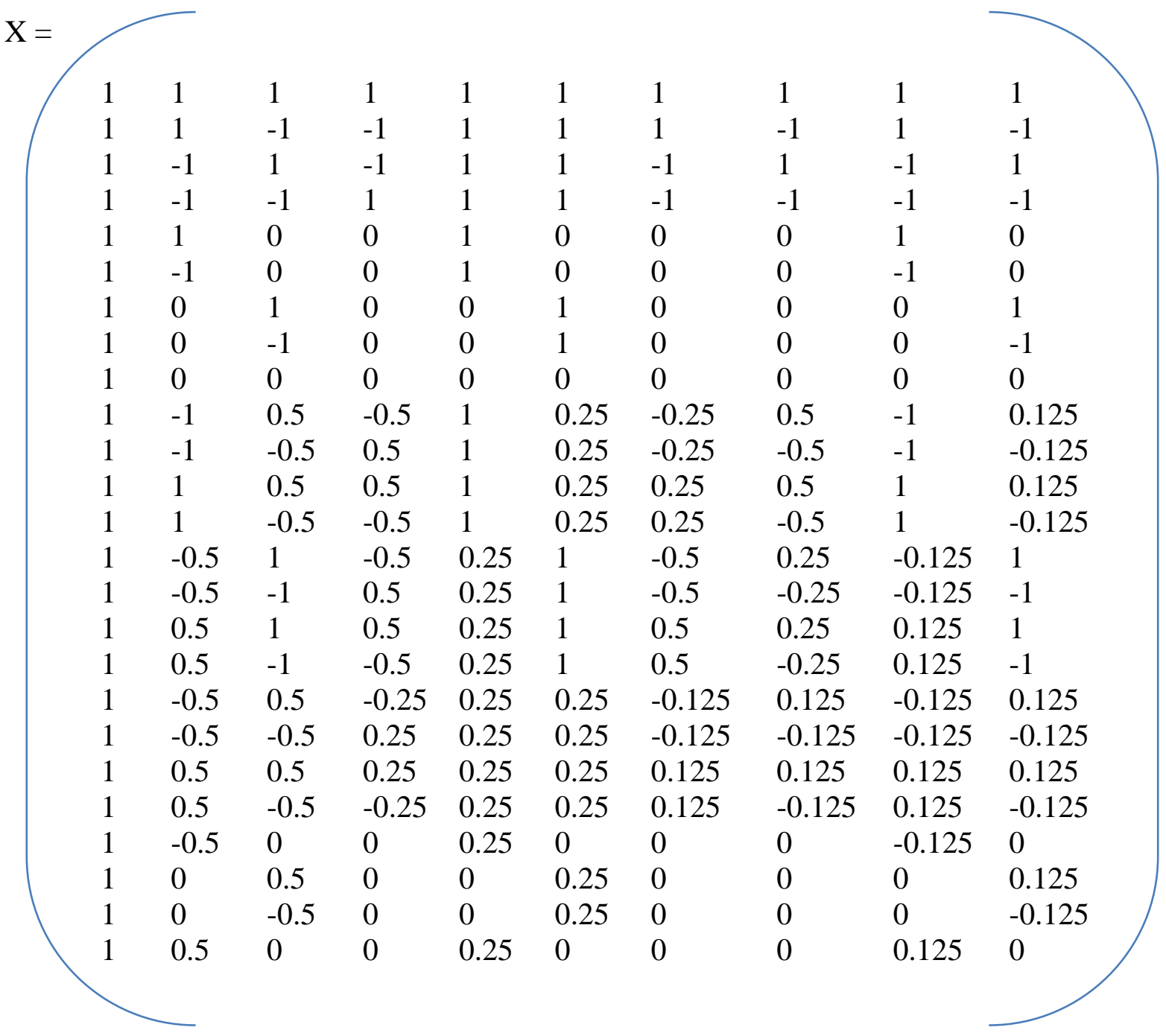


The associated hat matrix gives the elements (approximated to 4 decimal places) presented in the 25 columns below.

\section{Columns 1 through 16}

\begin{tabular}{|c|c|c|c|c|c|c|c|c|c|c|c|c|c|c|c|}
\hline 0.7429 & -0.0057 & -0.0057 & -0.1143 & 0.0257 & 0.0543 & 0.0257 & 0.0543 & -0.0743 & 0.0029 & 0.0486 & 0.2486 & -0.0257 & -0.0257 & 0.0486 & 0.2486 \\
\hline .0057 & 0.7429 & -0.1143 & -0.0057 & 0.0257 & 0.0543 & 0.0543 & 0.0257 & -0.0743 & 0.0486 & 0.0029 & -0.0257 & 0.2486 & 0.0486 & -0.0257 & 0.0029 \\
\hline-0.0057 & -0.1143 & 0.7429 & -0.0057 & 0.0543 & 0.0257 & 0.0257 & 0.0543 & -0.0743 & 0.2486 & -0.0257 & 0.0029 & 0.0486 & 0.2486 & 0.0029 & -0.0257 \\
\hline-0.1143 & -0.0057 & -0.0057 & 0.7429 & 0.0543 & 0.0257 & 0.0543 & 0.0257 & -0.0743 & -0.0257 & 0.2486 & 0.0486 & 0.0029 & 0.0029 & 0.2486 & 0.0486 \\
\hline 0.0257 & 0.0257 & 0.0543 & 0.0543 & 0.3686 & -0.0600 & -0.0743 & -0.0743 & 0.0400 & -0.0314 & -0.0314 & 0.2829 & 0.2829 & 0.0114 & 0.0114 & -0.1029 \\
\hline 0.0543 & 0.0543 & 0.0257 & 0.0257 & -0.0600 & 0.3686 & -0.0743 & -0.0743 & 0.0400 & 0.2829 & 0.2829 & -0.0314 & -0.0314 & -0.1029 & -0.1029 & 0.0114 \\
\hline 0.0257 & 0.0543 & 0.0257 & 0.0543 & $-\mathbf{- 0 . 0 7 4 3}$ & -0.0743 & 0.3686 & -0.0600 & 0.0400 & -0.1029 & 0.0114 & $-\mathbf{0 . 1 0 2 9}$ & 0.0114 & 0.2829 & -0.0314 & 0.2829 \\
\hline 0.0543 & 0.0257 & 0.0543 & 0.0257 & $-\mathbf{- 0 . 0 7 4 3}$ & -0.0743 & -0.0600 & 0.3686 & 0.0400 & 0.0114 & -0.1029 & 0.0114 & -0.1029 & -0.0314 & 0.2829 & $-\mathbf{0 . 0 3 1 4}$ \\
\hline-0.0743 & -0.0743 & -0.0743 & -0.0743 & 0.0400 & 0.0400 & 0.0400 & 0.0400 & 0.1543 & 0.0114 & 0.0114 & 0.0114 & 0.0114 & 0.0114 & 0.0114 & 0.0114 \\
\hline 0.0029 & 0.0486 & 0.2486 & -0.0257 & -0.0314 & 0.2829 & -0.1029 & 0.0114 & 0.0114 & 0.4086 & 0.0714 & 0.0714 & -0.1057 & -0.0343 & -0.0571 & -0.0571 \\
\hline 0.0486 & 0.0029 & -0.0257 & 0.2486 & -0.0314 & 0.2829 & 0.0114 & -0.1029 & 0.0114 & 0.0714 & 0.4086 & -0.1057 & 0.0714 & -0.0571 & -0.0343 & 0.0800 \\
\hline 0.2486 & -0.0257 & 0.0029 & 0.0486 & 0.2829 & -0.0314 & -0.1029 & 0.0114 & 0.0114 & 0.0714 & -0.1057 & 0.4086 & 0.0714 & -0.0571 & 0.0800 & $-\mathbf{0 . 0 3 4 3}$ \\
\hline-0.0257 & 0.2486 & 0.0486 & 0.0029 & 0.2829 & -0.0314 & 0.0114 & -0.1029 & 0.0114 & -0.1057 & 0.0714 & 0.0714 & 0.4086 & 0.0800 & -0.0571 & -0.0571 \\
\hline-0.0257 & 0.0486 & 0.2486 & 0.0029 & 0.0114 & -0.1029 & 0.2829 & -0.0314 & 0.0114 & -0.0343 & -0.0571 & -0.0571 & 0.0800 & 0.4086 & 0.0714 & 0.0714 \\
\hline 0.0486 & $-\mathbf{0 . 0 2 5 7}$ & 0.0029 & 0.2486 & 0.0114 & $-\mathbf{0 . 1 0 2 9}$ & -0.0314 & 0.2829 & 0.0114 & $-\mathbf{0 . 0 5 7 1}$ & $-\mathbf{0 . 0 3 4 3}$ & 0.0800 & -0.0571 & 0.0714 & 0.4086 & $\mathbf{- 0 . 1 0 5 7}$ \\
\hline 0.2486 & 0.0029 & $\mathbf{- 0 . 0 2 5 7}$ & 0.0486 & -0.1029 & 0.0114 & 0.2829 & $-\mathbf{0 . 0 3 1 4}$ & 0.0114 & -0.0571 & 0.0800 & -0.0343 & -0.0571 & 0.0714 & -0.1057 & 0.4086 \\
\hline 0.0029 & 0.2486 & 0.0486 & $-\mathbf{0 . 0 2 5 7}$ & -0.1029 & 0.0114 & -0.0314 & 0.2829 & 0.0114 & 0.0800 & -0.0571 & -0.0571 & -0.0343 & -0.1057 & 0.0714 & 0.0714 \\
\hline-0.0571 & 0.0800 & -0.0343 & -0.0571 & 0.0114 & 0.0686 & 0.0686 & 0.0114 & 0.0971 & 0.1457 & -0.0657 & 0.0771 & -0.0543 & 0.1457 & 0.0771 & -0.0657 \\
\hline $\mathbf{0 . 0 8 0 0}$ & $-\mathbf{0 . 0 5 7 1}$ & -0.0571 & -0.0343 & 0.0114 & 0.0686 & 0.0114 & 0.0686 & 0.0971 & -0.0657 & 0.1457 & -0.0543 & 0.0771 & 0.0771 & 0.1457 & -0.0543 \\
\hline-0.0343 & $-\mathbf{0 . 0 5 7 1}$ & $-\mathbf{0 . 0 5 7 1}$ & 0.0800 & 0.0686 & 0.0114 & 0.0686 & 0.0114 & 0.0971 & 0.0771 & -0.0543 & 0.1457 & -0.0657 & $-\mathbf{0 . 0 6 5 7}$ & $-\mathbf{0 . 0 5 4 3}$ & 0.1457 \\
\hline-0.0571 & $-\mathbf{0 . 0 3 4 3}$ & 0.0800 & -0.0571 & 0.0686 & 0.0114 & 0.0114 & 0.0686 & 0.0971 & $-\mathbf{0 . 0 5 4 3}$ & 0.0771 & $-\mathbf{0 . 0 6 5 7}$ & 0.1457 & $-\mathbf{0 . 0 5 4 3}$ & $-\mathbf{0 . 0 6 5 7}$ & 0.0771 \\
\hline 0.0114 & 0.0114 & -0.1029 & -0.1029 & 0.0114 & 0.1257 & 0.0114 & 0.0114 & 0.1257 & 0.0686 & 0.0686 & 0.0114 & 0.0114 & 0.0686 & 0.0686 & -0.0743 \\
\hline-0.1029 & 0.0114 & -0.1029 & 0.0114 & 0.0114 & 0.0114 & 0.1257 & 0.0114 & 0.1257 & 0.0686 & -0.0743 & 0.0686 & -0.0743 & 0.0686 & 0.0114 & 0.0686 \\
\hline 0.0114 & -0.1029 & 0.0114 & -0.1029 & 0.0114 & 0.0114 & 0.0114 & 0.1257 & 0.1257 & -0.0743 & 0.0686 & -0.0743 & 0.0686 & 0.0114 & 0.0686 & 0.0114 \\
\hline 0.1029 & $-\mathbf{0 . 1 0 2 9}$ & 0.0114 & 0.0114 & 0.1257 & 0.0114 & 0.0114 & 0.0114 & 0.1257 & 0.0114 & 0.0114 & 0.0686 & 0.0686 & -0.0743 & -0.0743 & 0.0686 \\
\hline
\end{tabular}




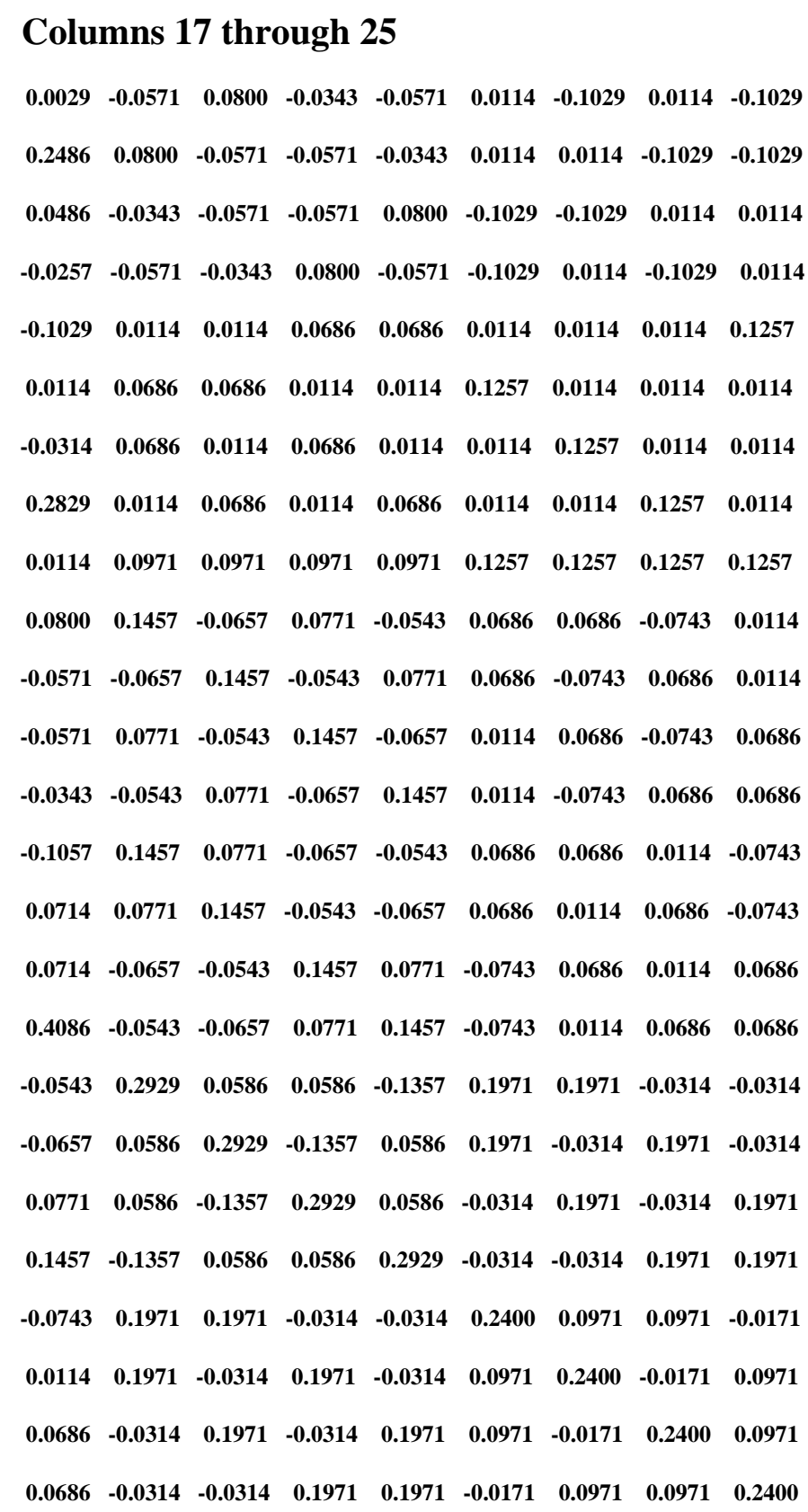

The diagonal elements $h_{i i}$ of the hat matrix and the respective candidate points are given in Table 4.1. The associated candidate points are grouped according to the magnitude of the diagonal elements and are presented in Table 4.2.

Table 4.1 The $h_{\mathrm{ii}}$ elements of the hat matrix for candidate points in two-variable case

\begin{tabular}{|c|c|c|c|}
\hline Candidate points & $\mathbf{X}_{\mathbf{1}}$ & $\mathbf{X}_{\mathbf{2}}$ & $\mathbf{h}_{\mathbf{i i}}$ \\
\hline 1 & 1 & 1 & 0.7428571 \\
2 & 1 & -1 & 0.7428571 \\
3 & -1 & 1 & 0.7428571 \\
4 & -1 & -1 & 0.7428571 \\
\hline
\end{tabular}




\begin{tabular}{|c|c|c|c|}
\hline 5 & 1 & 0 & 0.3685714 \\
6 & -1 & 0 & 0.3685714 \\
7 & 0 & 1 & 0.3685714 \\
8 & 0 & -1 & 0.3685714 \\
9 & 0 & 0 & 0.1542857 \\
10 & -1 & 0.5 & 0.4085714 \\
11 & -1 & -0.5 & 0.4085714 \\
12 & 1 & 0.5 & 0.4085714 \\
13 & 1 & -0.5 & 0.4085714 \\
14 & -0.5 & 1 & 0.4085714 \\
15 & -0.5 & -1 & 0.4085714 \\
16 & 0.5 & 1 & 0.4085714 \\
17 & 0.5 & -1 & 0.4085714 \\
18 & -0.5 & 0.5 & 0.2928571 \\
19 & -0.5 & -0.5 & 0.2928571 \\
20 & 0.5 & 0.5 & 0.2928571 \\
21 & 0.5 & -0.5 & 0.2928571 \\
22 & -0.5 & 0 & 0.24 \\
23 & 0 & 0.5 & 0.24 \\
24 & 0 & -0.5 & 0.24 \\
25 & 0.5 & 0 & 0.24 \\
\hline
\end{tabular}

Table 4.2 Summary of components of $h_{i i}$ and block arrangement in two variable case

\begin{tabular}{|c|c|c|c|}
\hline Blocks & $\begin{array}{c}\text { Design points for two } \\
\text { variables }\end{array}$ & $\mathbf{h}_{\mathbf{i i}}$ & Block size \\
\hline 1 & {$[ \pm 1, \pm 1]$} & 0.7428571 & 4 \\
2 & {$[ \pm 1, \pm 0.5],[ \pm 0.5, \pm 1]$} & 0.4085714 & 8 \\
3 & {$[ \pm 1,0],[0, \pm 1]$} & 0.3685714 & 4 \\
4 & {$[ \pm 0.5, \pm 0.5]$} & 0.2928571 & 4 \\
5 & {$[ \pm 0.5,0],[0, \pm 5]$} & 0.24 & 4 \\
6 & {$[0,0]$} & 0.1542857 & 1 \\
\hline
\end{tabular}

\section{Two-Variable Non-Sequential Design 1 (2VNSD-1):}

This design was generated using four blocks namely, blocks 1, 2, 3 and 4. A total of 20 experimental runs result from addition of the block since the generation of block size is a complete permutation of elements of a given design tuple. For instance, the block size of 4 associated with Block 3 is obtained using the arrangements $[ \pm 1,0]$ and $[0, \pm 1]$. The generated 20 -point design is as given below and has determinant value $6.366469 \times 10^{-8}$ of the normalized information matrix. 


$$
\xi_{20}=
$$

$\left(\begin{array}{ll}1 & 1 \\ 1 & -1 \\ -1 & 1 \\ -1 & -1 \\ 1 & 0 \\ -1 & 0 \\ 0 & 1 \\ 0 & -1 \\ -1 & 0.5 \\ -1 & -0.5 \\ 1 & 0.5 \\ 1 & -0.5 \\ -0.5 & 1 \\ -0.5 & -1 \\ 0.5 & 1 \\ 0.5 & -1 \\ -0.5 & 0.5 \\ -0.5 & -0.5 \\ 0.5 & 0.5 \\ 0.5 & -0.5 \\ 0 & \end{array}\right.$

\section{Two-Variable Non-Sequential Design 2 (2VNSD-2):}

In keeping with the size of the two-variable third-order designs of Yang (2008), a nonsequential design of size 17 was generated. The design points were chosen from Block 1 with block size of 4 , Block 2 with block size of 8 , Block 3 with block size of 4 and only one point from block 4. The generated 17-point design is as given below and has determinant value $4.575455 \times 10^{-8}$ of the normalized information matrix. 


$\xi_{17}=\left(\begin{array}{ll}1 & 1 \\ 1 & -1 \\ -1 & 1 \\ -1 & -1 \\ -1 & 0.5 \\ -1 & -0.5 \\ 1 & 0.5 \\ 1 & -0.5 \\ -0.5 & 1 \\ -0.5 & -1 \\ 0.5 & 1 \\ 0.5 & -1 \\ 1 & 0 \\ -1 & 0 \\ 0 & 1 \\ 0 & -1 \\ -0.5 & 0.5 \\ 0 & \end{array}\right)$

\section{Two-Variable Non-Sequential Design 3 (2VNSD-3):}

Another design with minimum run-size is generated with three blocks to give 16 experimental runs. The design points are formed using block $1 ;[ \pm 1, \pm 1]$, block $2 ;[ \pm 1, \pm 0.5]$ and block 3 ; $[ \pm 1,0]$. Associated $h_{i i}$ values of the hat matrix are $0.7428571,0.4085714$, and 0.3685714 respectively. The 16 point design and moment matrix are shown below.

$\xi_{16}=$

$\left(\begin{array}{cc}1 & 1 \\ 1 & -1 \\ -1 & 1 \\ -1 & -1 \\ -1 & 0.5 \\ -1 & -0.5 \\ 1 & 0.5 \\ 1 & -0.5 \\ -0.5 & 1 \\ -0.5 & -1 \\ 0.5 & 1 \\ 0.5 & -1 \\ 1 & 0 \\ -1 & 0 \\ 0 & 1 \\ 0 & -1\end{array}\right)$


The 16 point design gave a determinant value of $4.045144 \times 10^{-8}$ of the normalized information matrix.

Table 4.3 gives the Scaled Prediction Variance for the new non-sequential designs in two variables. The Scaled Prediction Variance is given as

$$
\mathrm{SPV}=N \underline{x}^{\prime}\left(X^{\prime} X\right)^{-1} \underline{x}=V[\underline{y}(\underline{x})]
$$

These values are essential in obtaining the G-efficiency values of the designs.

Table 4.3: Scaled Prediction Variance of the designs in two variables

\begin{tabular}{|c|c|c|c|c|c|c|c|}
\hline \multirow[b]{2}{*}{$\begin{array}{l}\text { Design } \\
\text { Points }\end{array}$} & \multirow[b]{2}{*}{ 2VNSD-1 } & \multirow[b]{2}{*}{ 2VNSD-2 } & \multirow[b]{2}{*}{ 2VNSD-3 } & \multicolumn{4}{|c|}{ Yang (2008) two-variable designs } \\
\hline & & & & FTOD & CCDA & LHDA & NFCD \\
\hline 1 & 15.8465 & 14.6996 & 14.2159 & 14.9616 & 16.0235 & 11.9889 & 16.1656 \\
\hline 2 & 15.8465 & 15.0578 & 14.2159 & 16.3119 & 16.0235 & 11.9889 & 16.1656 \\
\hline 3 & 15.8465 & 14.0819 & 14.2159 & 12.946 & 16.0235 & 11.9889 & 16.0761 \\
\hline 4 & 15.8465 & 14.6996 & 14.2159 & 14.6606 & 16.0235 & 11.9889 & 16.0761 \\
\hline 5 & 7.98469 & 8.01626 & 9.00803 & 9.70485 & 13.7585 & 8.18692 & 13.4433 \\
\hline 6 & 7.98469 & 9.47867 & 9.00803 & 10.8978 & 13.7585 & 8.18692 & 13.4433 \\
\hline 7 & 7.98469 & 8.89723 & 9.00803 & 10.5477 & 13.7585 & 8.18692 & 13.9378 \\
\hline 8 & 7.98469 & 9.32958 & 9.00803 & 9.92571 & 13.7585 & 8.18692 & 13.2772 \\
\hline 9 & 8.59571 & 8.01626 & 9.00803 & 13.4651 & 3.16949 & 3.65008 & 8.70347 \\
\hline 10 & 8.59571 & 8.89723 & 9.00803 & 13.2249 & 5.96281 & 15.127 & 8.70347 \\
\hline 11 & 8.59571 & 9.47867 & 9.00803 & 5.92433 & 5.96281 & 15.127 & 6.53332 \\
\hline 12 & 8.59571 & 9.32958 & 9.00803 & 6.23432 & 5.96281 & 15.127 & 6.53332 \\
\hline 13 & 8.59571 & 8.16964 & 7.76807 & 6.5157 & 5.96281 & 15.127 & 5.13149 \\
\hline 14 & 8.59571 & 7.43776 & 7.76807 & 6.76892 & 5.96281 & 6.28467 & 5.13149 \\
\hline 15 & 8.59571 & 7.43776 & 7.76807 & 5.75186 & 5.96281 & 6.28467 & 3.90834 \\
\hline 16 & 8.59571 & 8.16964 & 7.76807 & 5.92433 & 5.96281 & 6.28467 & 3.90834 \\
\hline 17 & 8.97741 & 8.80292 & & 6.23432 & 5.96281 & 6.28467 & 2.86195 \\
\hline 18 & 8.97741 & & & & & & \\
\hline 19 & 8.97741 & & & & & & \\
\hline 20 & 8.97741 & & & & & & \\
\hline
\end{tabular}




\section{Analysis involving three design variables}

For $k=3$, the full parameter response surface model is given as

$y=\beta_{0}+\beta_{1} x_{1}+\beta_{2} x_{2}+\beta_{3} x_{3}+\beta_{12} x_{1} x_{2}+\beta_{13} x_{1} x_{3}+\beta_{23} x_{2} x_{3}+\beta_{11} x_{1}^{2}+\beta_{22} x_{2}^{2}+$ $\beta_{33} x_{3}^{2}+\beta_{122} x_{1} x_{2}^{2}+\beta_{133} x_{1} x_{3}^{2}+\beta_{112} x_{11}^{2} x_{2}+\beta_{113} x_{11}^{2} x_{3}+\beta_{233} x_{2} x_{3}^{2}+\beta_{223} x_{22}^{2} x_{3}+\beta_{123} x_{1} x_{2} x_{3}$ $+\beta_{111} x_{1}^{3}+\beta_{222} x_{2}^{3}+\beta_{333} x_{3}^{3}+\varepsilon$

and contains 20 model parameters. The model matrix $X$ corresponding to the $5^{k}=125$ grid points allows for the computation of the hat matrix whose diagonal elements are shown in Table 4.5.

Table 4.5. Summary of components of $\boldsymbol{h}_{i i}$ and block arrangement in three-variable case

\begin{tabular}{|c|c|c|c|}
\hline Blocks & Design points for three variables & $\mathbf{h}_{\text {ii }}$ & Block size \\
\hline 1 & {$[ \pm 1, \pm 1, \pm 1]$} & 0.39942857 & 8 \\
\hline 2 & {$[ \pm 1, \pm 1, \pm 0.5],[ \pm 1, \pm 0.5, \pm 1],[ \pm 0.5, \pm 1, \pm 1]$} & 0.22628571 & 24 \\
\hline 3 & {$[ \pm 1, \pm 1,0],[ \pm 1,0, \pm 1],[0, \pm 1, \pm 1]$} & 0.20571429 & 12 \\
\hline 4 & {$[ \pm 1, \pm 0.5, \pm 0.5],[ \pm 0.5, \pm 1, \pm 0.5],[ \pm 0.5, \pm 0.5, \pm 1]$} & 0.13285714 & 24 \\
\hline 5 & {$[ \pm 1, \pm 0.5,0],[0, \pm 1, \pm 0.5],[ \pm 0.5,0, \pm 1]$} & 0.12171429 & 24 \\
\hline 6 & {$[ \pm 0.5, \pm 1,0],[0, \pm 0.5, \pm 1],[ \pm 1,0, \pm 0.5]$} & 0.108 & 6 \\
\hline 7 & {$[ \pm 1,0,0],[0, \pm 1,0],[0,0, \pm 1]$} & 0.09214286 & 8 \\
\hline 8 & {$[ \pm 0.5, \pm 0.5, \pm 0.5]$} & 0.08142857 & 12 \\
\hline 9 & {$[ \pm 0.5,0,0],[0, \pm 0.5,0],[0,0, \pm 0.5]$} & 0.06514286 & 6 \\
\hline 10 & {$[0,0,0]$} & 0.04228571 & 1 \\
\hline
\end{tabular}

The non-sequential design is generated using three blocks. The blocks are block $1 ;[ \pm 1, \pm 1$, $\pm 1]$, block $2 ;[ \pm 1, \pm 1, \pm 0.5],[ \pm 1, \pm 0.5, \pm 1],[ \pm 0.5, \pm 1, \pm 1]$ and block $6 ;[ \pm 1,0,0],[0, \pm 1,0]$ ,$[0,0, \pm 1]$. The associated diagonal element $h_{\mathrm{ii}}$ values of the hat matrix are 0.39942857 , 0.22628571 and 0.108 respectively. This gives a 38-point design with a determinant value of $2.192577 \times 10^{-15}$ of the normalized information matrix.

The 38-point three-variable non-sequential design (3VNSD) is as follows; 
African Journal of Mathematics and Statistics Studies

ISSN: 2689-5323

Volume 5, Issue 1, 2022 (pp. 14-32)

www.abjournals.org

$\xi_{38}=$

\begin{tabular}{|c|c|c|}
\hline-1 & -1 & -1 \\
\hline 1 & -1 & -1 \\
\hline-1 & 1 & -1 \\
\hline 1 & 1 & -1 \\
\hline-1 & -1 & 1 \\
\hline 1 & -1 & 1 \\
\hline-1 & 1 & 1 \\
\hline 1 & 1 & 1 \\
\hline-1 & -1 & -0.5 \\
\hline-1 & -1 & 0.5 \\
\hline 1 & 1 & -0.5 \\
\hline 1 & 1 & 0.5 \\
\hline-1 & -0.5 & -1 \\
\hline-1 & -0.5 & 1 \\
\hline 1 & 0.5 & -1 \\
\hline 1 & 0.5 & 1 \\
\hline-1 & 0.5 & -1 \\
\hline-1 & 0.5 & 1 \\
\hline 1 & -0.5 & -1 \\
\hline 1 & -0.5 & 1 \\
\hline-1 & 1 & -0.5 \\
\hline-1 & 1 & 0.5 \\
\hline 1 & -1 & -0.5 \\
\hline 1 & -1 & 0.5 \\
\hline-0.5 & -1 & -1 \\
\hline-0.5 & -1 & 1 \\
\hline 0.5 & 1 & -1 \\
\hline 0.5 & 1 & 1 \\
\hline-0.5 & 1 & -1 \\
\hline-0.5 & 1 & 1 \\
\hline 0.5 & -1 & -1 \\
\hline 0.5 & -1 & 1 \\
\hline-1 & 0 & 0 \\
\hline 1 & 0 & 0 \\
\hline 0 & -1 & 0 \\
\hline 0 & 1 & 0 \\
\hline 0 & 0 & -1 \\
\hline 0 & 0 & 1 \\
\hline
\end{tabular}


The Scaled Prediction Variances of the three-variable designs are summarized in Table 4.6.

Table 4.6. Summary of the Scaled Prediction Variance (SPV) for all designs in three variables.

\begin{tabular}{|c|c|}
\hline Design Points & 3VNSD \\
\hline 1 & 21.54793 \\
\hline 2 & 21.54793 \\
\hline 3 & 21.54793 \\
\hline 4 & 21.54793 \\
\hline 5 & 21.54793 \\
\hline 6 & 21.54793 \\
\hline 7 & 21.54793 \\
\hline 8 & 21.54793 \\
\hline 9 & 19.34076 \\
\hline 10 & 19.34076 \\
\hline 11 & 19.34076 \\
\hline 12 & 19.34076 \\
\hline 13 & 19.34076 \\
\hline 14 & 19.34076 \\
\hline 15 & 19.34076 \\
\hline 16 & 19.34076 \\
\hline 17 & 19.34076 \\
\hline 18 & 19.34076 \\
\hline 19 & 19.34076 \\
\hline 20 & 19.34076 \\
\hline 21 & 19.34076 \\
\hline 22 & 19.34076 \\
\hline 23 & 19.34076 \\
\hline 24 & 19.34076 \\
\hline 25 & 19.34076 \\
\hline 26 & 19.34076 \\
\hline 27 & 19.34076 \\
\hline 28 & 19.34076 \\
\hline 29 & 19.34076 \\
\hline 30 & 19.34076 \\
\hline 31 & 19.34076 \\
\hline 32 & 19.34076 \\
\hline 33 & 20.57307 \\
\hline 34 & 20.57307 \\
\hline 35 & 20.57307 \\
\hline 36 & 20.57307 \\
\hline 37 & 20.57307 \\
\hline 38 & 20.57307 \\
\hline
\end{tabular}




\section{G-efficiencies of the generated designs}

G-efficiency of a given design evaluates the maximum value of the Scaled Prediction Variance (SPV) of a design in reference to its theoretical minimum scaled prediction variance whose value equals the number of model parameters. The G-efficiency of a design is computed using the expression

$\mathrm{G}-\mathrm{eff}=\frac{p}{\operatorname{Max} S P V}$

where $p$ is the number of model parameter and Max SPV is the maximum SPV of the design.

Table 4.3. Summary of G-efficiencies of the designs

\begin{tabular}{|c|c|c|c|c|c|c|c|c|}
\hline Design & 2VNSD-1 & 2VNSD-2 & 2VNSD-3 & FTOD & CCDA & LHDA & NFCD & 3VNSD \\
\hline MaxSPV & 15.0578 & 14.2159 & 15.8465 & 16.3119 & 16.0235 & 15.1270 & 16.1656 & 21.54793 \\
\hline $\mathrm{N}$ & 20 & 17 & 16 & 17 & 17 & 17 & 17 & 38 \\
\hline$p$ & 10 & 10 & 10 & 10 & 10 & 10 & 10 & 20 \\
\hline G-efficiency & $66.41 \%$ & $70.34 \%$ & $63.11 \%$ & $61.30 \%$ & $62.41 \%$ & $66.11 \%$ & $61.86 \%$ & $92.82 \%$ \\
\hline
\end{tabular}

\section{CONCLUSION}

New non-sequential third-order response surface designs have been generated using information contained in the hat matrix for given candidate points. The non-sequential designs are built from scratch but importance of the axial points is upheld. Specifically, the axial or star points play a vital role in the estimation of pure quadratic terms hence its inclusion provided for superior designs. Therefore all the new designs generated had the axial/star points included. When considering the scaled prediction variances of the new designs, the superiority of the new designs were also obvious as they show better predictive abilities than some of the existing designs. This superiority has been summarized using the G-efficiency index. The design points that make up the designs are easy to select since a continuous design space can be made discrete by grid formation. The new designs provide reasonable distribution of design points throughout the region of interest. The designs further provide precise estimates of model coefficients and offer good prediction variances over the experimental region. It is further interesting to note that the designs do not require too many experimental runs and further gain run size efficiency over some existing designs without losing their optimality efficiency.

\section{REFERENCES}

Alaeddini, A., Yang, K., Murat, A. (2013b). ASRSM: A Sequential Experimental Design for Response Surface Optimization. Quality and reliability Engineering International, 29(2): 241-258.

Anjaneyulu, G.V.S.R., Dattatreya Rao, A.V. and Narasimham, V.L. (2001). Third Order Slope 
Anjaneyulu, G.V.S.R., Dattatreya Rao, A.V. and Narasimham, V.L. (1994 - 1995) Construction of third order slope rotatable designs , Gujarat Statistical Review Vols.21 $\& 22,49-53$

Arshad, H. M., Akhtar, M. and Gilmour, S. G. (2012). Augmented Box-Behnken designs for fitting third order response surfaces. Communications in Statistics - Theory and Methods 41(23): 4225-4239.

Baker, F. D. and Bargmann, R. E. (1985). Orthogonal Central Composite Designs of the Third Order in the Evaluation of Sensitivity and Plant Growth Simulation Models. Journal of the American Statistical Association, Vol. 80. No. 391391, pp. 574-579.

Bose, R. C. and Draper, N. R. (1958). Rotatable designs of second and third order in three or more dimensions. Institute of Statistics, Mimeograph Series No. 197.

Box, G. E. P. (1952). Multifactor designs of first order. Biometrika, 39:49-57.

Box, G. E. P. (1954). The exploration and exploitation of response surfaces 8 some general considerations and examples. Biometrics, 10:16-60.

Box, G. E. P. and Behnken, D. W. (1959). Simplex-Sum Designs a Class of Second Order Rotatable Designs Derivable From Those of First Order, Institute of Statistics Mimeograph Series No. 232.

Box, G. E. P. and Behnken, D. W. (1960). Some new three level designs for the study of quantitative variables. Technometrics, 2, 455-475. http://dx.doi.org/10.1080/00401706.1960.10489912

Box, G. E. P., and Wilson, K. B. (1951). On the Experimental Attainment of Optimum Conditions. Journal of the Royal Statistical Society, 13, 1-45.

Castillo, F.A., J.D. Sweeney, J.D. and Zirk, W.E. (2004). Using evolutionary algorithms to suggest variable transformations in linear model lack of fit situations. In Proceedings of the Congress on Evolutionary Computations, pp. 556-560. https://ieeexplore.ieee.org/document/1330906

Cheng, S-W. and Wu, C. F. J. (2001). Factor Screening And Response Surface Exploration. Statistica Sinica 11: 553-604.

Das, M.N. (1961) Construction of rotatable designs from factorial designs, Journal of Indian Society for Agriculture Statistics.,Vol.13,169-194.

Das, M.N. and Narasimham, V.L. (1962). Construction of rotatable designs through balanced incomplete block designs, Annals of Mathematical Statistics, Vol.33,1421-1439.

Dette, H and Kwiecien, R. (2004). A comparison of sequential and non-sequential designs for discrimination between nested regression models, Biometrika, 91(1): 165-176.

Dette, H. and Grigoriev, Y. (2014). E-Optimal Designs For Second-Order Response Surface Models. The Annals of Statistics, Vol. 42, No. 4, 1635-1656, DOI: 10.1214/14AOS1241.

Draper, N.R. (1960a). "Third order rotatable designs in three dimensions." Annals of Mathematical Statistics 31: 865-874.

Draper, N.R. (1960b). "A third order rotatable design in four dimensions. "Annals of Mathematical statistics 31:875-877

Eighty Points in Four Dimensions with a Hypothetical Case Study. Asian Journal of Probability and Statistics 4(4): 1-9.

Erickson, G. E. (2007). Overview of Supersonic Aerodynamics Measurement Techniques in the NASA Langley Unitary Plan Wind Tunnel. Washington, DC: NASA.

Gao, G-M., Zou, H-F, Liu, D-R, Miao, L-N., Gan, S-C., An, B-C., Xu, J-J. and Li, G-H. (2009). Synthesis of ultrafine silica powders based on oil shale ash by fluidized bed drying of wet-gel slurry. Fuel 88(7): 1223-7. 
Gardiner, D. A., Grandage, A. H. E. and Hader, R. J. (1959). Third order rotatable designs for exploring response surface, The Annals of Mathematical Statistics, Volume 30, pp, $1082-$

Kahng, M. W. (2007). Leverages measures in Nonlinear Regression, Journal of Korean Data \& Information Science Society, 18(1): 229-235.

Khuri, A. I. (2017). A General Overview of Response Surface Methodology. Biom Biostat Int J 5(3): 00133. DOI: 10.15406/bbij.2017.05.00133.

Landman, D., Simpson, J., Mariani, R., Ortiz, F., and Britcher, C. (2007). Hybrid Design for Aircraft Wind-Tunnel Testing Using Response Surface Methodologies. Journal of Aircraft, Vol.44, No. 4.

Mutiso, J.M. (1998)."Second and third order specific and sequential rotatable designs in k dimensions".D.Phil. Thesis, Moi university, Eldoret Kenya

Myers, R. H., Montgomery, D. C. and Anderson-Cook, C. M. (2009). Response Surface Methodology, Process And Product Optimization Using Designed Experiments. Wiley, New York, NY.

Norulaini, N. A. N., Setiano, W. B., Zaidul, I. S. M., Nawi, A. H., Azizi, C. Y.M., and Omar, A. K. M. (2009). Effects of supercritical carbon dioxide extraction parameters on virgin coconut oils yields and medium-chain triglyceride content. Food Chemistry 116(1): 193-197.

Nyakundi, O. C. (2019). A hypothetical sequential third Order rotatable design in five Dimensions. European International Journal of Science and Technology, 8(4), 40-50.

Nyakundi, O. C. and Matunde, N. C. (2019). A Sequential Third Order Rotatable Design of rotatable designs using BIBD, International Review of Applied Engineering Research. Volume 4, Number 1, pp. 89-96.

Rotatable Designs, Journal of Indian Society for Probability and statistics,. Vol.5,75-79.

Sanchez S. M. and Sanchez P. J. (2005). Very large fractional factorial and central composite designs. ACM Transactions on Modeling and Computer Simulation (TOMACS) 2005; 15(4):362-377.

Seshu Babu, P., Dattatreya Rao, A. V., Anjaneyulu, G. V. S. R. and Srinivas, K. (2015). Cubic Response Surface Designs Using Bibd In Four Dimensions. Applied Mathematics and Sciences: An International Journal, Vol. 2, No. 1, pp. 17-25.

Seshu Babu, P., Dattatreya Rao, A.V. and Srinivas K.(2014). Construction of third order slope

Yang, Y. (2008). Multiple Criteria Third-Order Response Surface Design and Comparison. MSc Dissertation submitted to FAMU-FSU College of Engineering, Florida State University. 